\title{
Une organisation maternaliste au Québec la Fédération nationale Saint-Jean-Baptiste et la bataille pour le vote des femmes
}

\section{Karine Hébert}

Volume 52, numéro 3, hiver 1999

URI : https://id.erudit.org/iderudit/005467ar

DOI : https://doi.org/10.7202/005467ar

Aller au sommaire du numéro

Éditeur(s)

Institut d'histoire de l'Amérique française

ISSN

0035-2357 (imprimé)

1492-1383 (numérique)

Découvrir la revue

Citer cet article

Hébert, K. (1999). Une organisation maternaliste au Québec la Fédération nationale Saint-Jean-Baptiste et la bataille pour le vote des femmes. Revue d'histoire de l'Amérique française, 52(3), 315-344.

https://doi.org/10.7202/005467ar
Résumé de l'article

Cet article présente, à la lumière des développements récents de l'historiographie féministe, une nouvelle interprétation de la Fédération nationale Saint-Jean-Baptiste. Cette réinterprétation se base sur un concept assez nouveau: le maternalisme, défini comme une catégorie d'analyse utilisée pour qualifier les mouvements de femmes chez qui la maternité constituait le point central de l'idéologie et de l'argumentation. Nous examinons, entre autres, le modèle féminin véhiculé par la FNSJB pour constater qu'il correspondait au modèle traditionnel de « la femme ", celui qui effectuait une équation entre la femme et la mère. La maternité n'était toutefois pas perçue par la FNSJB comme une fonction limitative, mais bien comme une ouverture vers la société. Elle a donc dû redéfinir les frontières entre les sphères privée et publique pour permettre aux femmes de transgresser celles du foyer et faire bénéficier la société de leur influence maternelle. Le cadre d'analyse élaboré dans la première partie est ensuite appliqué à un cas concret de revendication: la bataille pour le vote des femmes.
Tous droits réservés @ Institut d'histoire de l'Amérique française, 1999
Ce document est protégé par la loi sur le droit d'auteur. L'utilisation des services d’Érudit (y compris la reproduction) est assujettie à sa politique d'utilisation que vous pouvez consulter en ligne. 


\title{
UNE ORGANISATION MATERNALISTE AU QUÉBEC LA FÉDÉRATION NATIONALE SAINT-JEAN-BAPTISTE ET LA BATAILLE POUR LE VOTE DES FEMMES ${ }^{1}$
}

\author{
KARINE HÉBERT \\ Département d'histoire \\ Université du Québec à Montréal
}

\section{RÉSUMÉ}

Cet article présente, à la lumière des développements récents de l'historiographie féministe, une nouvelle interprétation de la Fédération nationale Saint-Jean-Baptiste. Cette réinterprétation se base sur un concept assez nouveau: le maternalisme, défini comme une catégorie d'analyse utilisée pour qualifier les mouvements de femmes chez qui la maternité constituait le point central de l'idéologie et de l'argumentation. Nous examinons, entre autres, le modèle féminin véhiculé par la FNSJB pour constater qu'il correspondait au modèle traditionnel de «la femme», celui qui effectuait une équation entre la femme et la mère. La maternité n'était toutefois pas perçue par la FNSJB comme une fonction limitative, mais bien comme une ouverture vers la société. Elle a donc dû redéfinir les frontières entre les sphères privée et publique pour permettre aux femmes de transgresser celles du foyer et faire bénéficier la société de leur influence maternelle. Le cadre d'analyse élaboré dans la première partie est ensuite appliqué à un cas concret de revendication: la bataille pour le vote des femmes.

\section{ABSTRACT}

In the light of recent developments in feminist historiography, this article presents a new interpretation of the Fédération nationale Saint-Jean-Baptiste. This reinterpretation is based on a fairly new concept: maternalism, which is used to describe some women's movements for whom maternity constituted the central point of their ideology and argumentation. We first look at the feminine model conveyed by the FNSJB and then note its equivalence with the traditionnal model of «woman», which identified «woman» as «mother». Nonetheless, for the FNSJB maternity was not conceived as a limitative

1. Cet article reprend les grandes lignes du mémoire de maîtrise que j'ai présenté au département d'histoire de l'Université de Montréal en 1997, Une organisation maternaliste au Québec, la Fédération nationale Saint-Jean-Baptiste (1900-1940). Cette recherche a bénéficié de l'appui du Fonds FCAR. Je tiens à remercier Denyse Baillargeon, Fernande Roy et Julien Goyette qui ont lu et commenté la première version de cet article, ainsi que les évaluateurs anonymes de la Revue d'histoire de l'Amérique française qui m'ont fait de judicieuses suggestions. 
function but rather as an opening onto society. Thus, the FNSJB had to redefine the frontiers between public and private spheres, so that society could benefit from women's maternal influence. The framework of analysis developed in the first part is then applied to a case: the struggle for women's suffage.

De l'avis même de ses fondatrices, la Fédération nationale SaintJean-Baptiste avait pour objectif de «grouper les Canadiennes françaises catholiques en vue de fortifier par l'union leur action dans la famille et dans la société ${ }^{2}$. Pour la première fois, les Canadiennes françaises, particulièrement celles de Montréal, eurent l'occasion de participer à des œuvres dont le mandat dépassait la philanthropie. Cette nouvelle perspective d'action a été associée, jusqu'à aujourd'hui, aux luttes féministes. Un élargissement des interrogations et du vocabulaire en histoire des femmes et du féminisme, amorcé récemment aux États-Unis, m'incite toutefois à remettre en question l'utilisation du qualificatif féministe auquel on a eu recours pour décrire la FNSJB. Le maternalisme, nouvelle catégorie d'interprétation en études féministes, pourrait, à cet égard, permettre de mieux comprendre et mieux contextualiser cette organisation de femmes.

En remettant en question le féminisme de la FNSJB, je ne veux pas minimiser l'importance de cette organisation féminine dans la prise de conscience collective et individuelle des femmes du Québec au sujet de leur place dans la société ou encore dans l'amélioration de leurs conditions de vie. Il m'importait plutôt d'examiner comment, à la lumière des développements récents de l'historiographie féministe, la Fédération peut maintenant être comprise, 23 ans après les premières études de Yolande Pinard à ce sujet ${ }^{3}$. C'est donc en revisitant des sources connues, les archives de la FNSJB, celles de Marie Gérin-Lajoie, ainsi que La Bonne parole, que j'ai entrepris cette réinterprétation.

\section{QU'EST-CE QUE LE MATERNALISME?}

Ce n'est que tout dernièrement, au début des années 1990, que le concept de maternalisme a été diffusé par différents travaux en sociologie, en science politique et en histoire des femmes. Il faut replacer son émergence dans l'évolution récente du féminisme qui, depuis la fin des années

2. «La Fédération nationale Saint-Jean-Baptiste veut avoir sa maison», La Bonne parole (ciaprès $B P$ ), 11,12 (décembre 1923): 4.

3. En histoire féministe, dont je ne peux m'exclure, les «historiographical shifts have owed more to debates in feminist theory and politics since 1970 than to attributes inherent in the relevant historical evidence». Voir Judith Allen, «Contextualising Late-Nineteenth-Century Feminism: Problems and Comparisons», Journal of the Canadian Historical Association/Revue de la Société historique du Canada, nouvelle série, 1 (1990): 19. 
1980, est entre autres marqué par une individualisation des revendications ${ }^{4}$. La popularité croissante du postmodernisme, aux ÉtatsUnis notamment, a fait éclater le sujet féminin et entraîné l'émergence de l'histoire du genre ${ }^{5}$; les dimensions d'ethnie, de religion, d'origine sociale, d'orientation politique ou sexuelle s'ajoutent à la définition des sujets. En tenant compte de ces identités multiples, des chercheuses comme Nancy $\mathrm{Cott}^{6}$ en sont venues à remettre en question l'interprétation féministe qui dominait jusqu'alors dans l'étude du militantisme féminin du début du siècle. Plusieurs de ces nouveaux travaux s'inscrivent, en outre, dans la vague actuelle des recherches sur l'État et, plus spécialement, sur l'Étatprovidence. Les deux tendances, l'éclatement des sujets et les recherches sur l'État, se sont finalement rejointes pour mener à une réinterprétation des luttes des femmes du début du siècle. De là est né le concept de maternalisme, dans le but avoué d'affiner la perception qu'on avait de ces revendications féminines et d'analyser le rôle joué par les femmes dans le développement de l'État ${ }^{7}$. En effet, on a constaté que les groupes de femmes qui militaient au début du $\mathrm{XX}^{\mathrm{e}}$ siècle le faisaient souvent au nom de leur maternité potentielle et qu'elles revendiquaient des droits sociaux et politiques pour l'accomplissement du rôle que leur conférait cette potentialité.

Le maternalisme peut être brièvement défini comme une catégorie d'analyse utilisée pour qualifier certains mouvements féminins du début du siècle, à savoir ceux qui, justement, se caractérisaient par la place fondamentale de la maternité dans leur vision du monde et par l'utilisation de la maternité dans leur argumentation. Les femmes engagées dans ces mouvements souhaitaient élargir le champ des responsabilités qu'elles

4. Il serait intéressant d'élargir le propos et de situer le maternalisme dans l'évolution globale du féminisme et de l'histoire des femmes. J'ai approfondi cet aspect dans le premier chapitre de mon mémoire, Une organisation maternaliste au Québec. La Fédération nationale Saint-Jean-Baptiste (1900-1940).

5. Pour avoir une meilleure idée du débat existant actuellement entre les partisanes de l'histoire des femmes et celles de l'histoire du genre, on peut consulter les deux articles suivants: Joy Parr, «Gender History and Historical Practice», Canadian Historical Review, 76,3 (1995): 354-376; et Joan Sangster, «Beyond Dichotomies. Re-Assessing Gender History and Women's History in Canada», Left History, 3,1 (1995): 109-121. Par ailleurs, l'ensemble des travaux de Joan Scott sont assez représentatifs de l'histoire du genre et constituent, en outre, un exemple intéressant d'intégration de l'histoire du genre et de l'histoire politique et des idéologies. Voir Joan Scott, Gender and the Politics of History (New York, Columbia University Press, 1988).

6. Nancy Cott, «What's in a Name? The Limits of Social Feminism; Or Expanding the Vocabulary of Women's History», Journal of American History, 76 (décembre 1989): 809-829.

7. Mentionnons Seth Koven et Sonya Michel, Mother of a New World. Maternalist Politics and the Origins of Welfare States (New York, Routledge, 1993); Gisela Bock et Pat Thane, dir., Maternity and Gender Policies. Women and the Rise of European Welfare States, 1880-1950 (Londres, Routledge, 1994). 
détenaient déjà dans la famille à l'ensemble de la société. En fait, le maternalisme permet de distinguer les groupes de femmes pour qui la maternité constituait une priorité idéologique, de ceux qui niaient ou ignoraient cette réalité physique des femmes pour plutôt mettre l'accent sur une recherche de l'égalité. Jusqu'à maintenant, ces deux groupes, malgré une différence fondamentale dans l'argumentation et les priorités idéologiques, étaient désignés par le terme féministe auquel s'ajoutaient ensuite des qualificatifs: on parlait ainsi fréquemment de féminisme chrétien, de féminisme social, de féminisme maternel ou encore de féminisme égalitaire. Actuellement, plusieurs distinguent le féminisme et le maternalisme en insistant sur leur opposition argumentative ainsi qu'en faisant ressortir la dichotomie égalité/différence qui émane de leur discours. C'est ainsi que le féminisme, comme le mentionne Molly Ladd-Taylor, serait caractérisé par la recherche de l'égalité entre les hommes et les femmes, de même que par le rejet de la différence fondée sur la vocation maternelle des femmes ${ }^{8}$. De son côté, Nancy Cott définit le féminisme comme étant un mouvement oppositionnel et révolutionnaire ${ }^{9}$. Comme l'a résumé Linda Gordon: «Feminism is a critique of male supremacy, formed and offered in the light of a will to change it, which in turn assumes a conviction that it is changeable ${ }^{10} . »$ Le maternalisme, quant à lui, fonde ses revendications sur l'acceptation de la nature maternelle des femmes qui leur confère un rôle social particulier. Aussi hisse-t-il au premier rang du discours les concepts de différence et de complémentarité. La maternité, élément majeur et difficilement rejetable de l'identité féminine à l'époque, est présentée comme une justification des revendications sociales et comme un facteur d'unification des femmes au-delà des classes sociales, de la religion et de l'appartenance ethnique. Dans la pratique toutefois, féministes et maternalistes se rejoignaient parfois dans la revendication de certains droits, le droit de vote par exemple ou encore l'accès à l'éducation et au marché du travail. Seth Koven et Sonya Michel ont été les premiers à proposer une définition du maternalisme:

We apply the term to ideologies that exalted women's capacity to mother and extended to society as a whole the values of care, nurturance, and morality. Maternalism always operated on two levels: it extolled relationships to politics and the state, to community, workplace, and marketplace. In practice, maternalist

8. Molly Ladd-Taylor, Mother-Work. Women, Child Welfare, and the State, 1890-1930 (Urbana, University of Illinois Press, 1994), 104-105.

9. Nancy Cott, «What's in a Name?...», 828.

10. Définition utilisée par Nancy Cott, dans The Grounding of Modern Feminism (New Haven and London, Yale University Press, 1987), 4-5. 
ideologies often challenged the constructed boundaries between public and private, women and men, state and civil society ${ }^{11}$.

Depuis cette définition pionnière parue en 1990, d'autres chercheurs - et surtout des chercheuses - se sont penchés sur le sujet. Parmi ceuxlà, une historienne américaine, Molly Ladd-Taylor, s'est attardée à caractériser les différents mouvements féminins américains de la fin du XIX siècle et du début du $\mathrm{XX}^{\mathrm{e}}$ siècle. Selon elle, il faut distinguer trois principaux mouvements: le féminisme, le maternalisme progressiste et le maternalisme sentimental. Visible surtout à partir de l'obtention du droit de vote des femmes au début des années 1920, le féminisme américain rejetait l'argument maternel dans la quête d'une égalité complète des hommes et des femmes. Les maternalistes de toutes tendances, pour leur part, ont continué à faire de la maternité la pierre angulaire de leur argumentation. Les tenantes du maternalisme progressiste, au nom de la maternité potentielle des femmes, ont amorcé en partie le mouvement réformiste américain. Elles soutenaient que la capacité de maternage des femmes leur imposait certaines responsabilités envers les enfants, notamment, et que ces responsabilités nécessitaient un engagement accru des femmes dans le monde public et une réforme de celui-ci. Les maternalistes sentimentales sont ensuite définies par Ladd-Taylor comme les gardiennes du rôle traditionnel des femmes dans la société, c'est-à-dire que leur timide acceptation de l'extension des responsabilités maternelles des femmes à l'extérieur de leur foyer n'était pas, chez elles, synonyme d'une modification de leur statut social et juridique ni d'une redéfinition du rôle de l'État. L'étude de Ladd-Taylor est novatrice, car elle repose sur une réflexion structurée à propos des motivations qui ont amené les femmes du début du siècle à utiliser la maternité dans leur définition d'ellesmêmes et de leur place dans la société. Par contre, l'historienne se limite à appliquer le concept général de maternalisme aux revendications touchant le bien-être des mères et des enfants. Il serait intéressant de voir dans quelle mesure la maternité a pu être utilisée dans l'ensemble des revendications des femmes de cette époque ${ }^{12}$.

En étudiant la FNSJB selon le nouvel éclairage du maternalisme, j'endosse les grandes lignes proposées par Koven et Michel en 1990,

11. Seth Koven et Sonya Michel, «Womanly Duties: Maternalist Politics and the Origins of Welfare States in France, Germany, Great Britain, and the United States, 1880-1920», American Historical Review, 4 (octobre 1990): 1079.

12. C'est ce que je fais dans le troisième chapitre de mon mémoire lorsque j'étudie comment le maternalisme constitue la base argumentative et idéologique d'un certain nombre de dossiers défendus par la FNSJB: le suffrage féminin, la réforme du code civil, l'éducation, le travail salarié des femmes et la protection maternelle et infantile. 
reprises et raffinées par Ladd-Taylor dans son essai de catégorisation des mouvements féminins américains. C'est-à-dire que l'ampleur de la divergence idéologique et argumentative entre l'adhésion des mouvements féminins à la différence ou à l'égalité nécessite, à mon avis, une analyse qui en tienne compte de façon satisfaisante. Dans cette perspective, le maternalisme permet d'affiner la compréhension des mouvements de femmes québécoises de la première moitié du $\mathrm{XX}^{\mathrm{e}}$ siècle, ainsi que d'en saisir toute la diversité. On pourrait ainsi avancer que les Cercles des fermières constituaient un bon exemple, dans le contexte québécois, de ce qu'il convient d'appeler le maternalisme sentimental. Par ailleurs, le recours à ce concept s'insère dans une tentative, déjà réclamée par Joan Scott et Louise Toupin ${ }^{13}$, de sortir des analyses trop souvent essentialistes du féminisme actuel et de se débarrasser du carcan imposé par la dichotomie égalité/différence. En montrant le paradoxe des revendications de la FNSJB - qui demandait une égalité de droit sans faire appel à une égalité des sexes mais plutôt en se basant sur la notion de différence - je démontre les limites du cadre idéologique auquel les mouvements de femmes ont été confrontés jusqu'à aujourd'hui. L'impossibilité du choix entre le recours à l'égalité et à la différence ${ }^{14}$ est efficacement mise en lumière par le maternalisme qui réclamait la première en insistant sur la seconde.

\section{LA FÉDÉRATION NATIONALE SAINT-JEAN-BAPTISTE}

La FNSJB, première organisation de femmes catholiques canadiennes-françaises à voir le jour à Montréal en 1907, a déjà fait l'objet de quelques recherches et son histoire est assez bien connue ${ }^{15}$. Il est toutefois utile de rappeler qu'elle est née du désir de quelques Canadiennes françaises catholiques de se doter d'un lieu de rassemblement similaire à celui que possédaient déjà les anglophones protestantes, le Montreal Local

13. Joan W. Scott, «Deconstructing Equality-Versus-Difference or the Uses of Poststucturalist Theory for Feminism», Feminist Studies, 14,1 (1988): 33-50. Louise Toupin, «Une histoire du féminisme est-elle possible?», Recherches féministes, 6,1 (1993): 25-51.

14. Joan W. Scott, «Deconstructing Equality-Versus-Difference», 43.

15. Yolande Pinard, Marie Lavigne et Jennifer Stoddard, «La Fédération nationale SaintJean-Baptiste au début du $20^{\mathrm{e}}$ siècle», dans Marie Lavigne et Yolande Pinard, dir., Les femmes dans la société québécoise (Montréal, Boréal Express, 1977), 89-108; et «The Fédération nationale SaintJean-Baptiste and the Women's Movement in Québec», dans Linda Kealey, dir., A Not Unreasonable Claim (Toronto, Women's Educational Press, 1979), 71-87; Monique Dumais, La mère dans la société québécoise. Étude éthique d'un modèle à partir de deux journaux féministes: La Bonne Parole (1913-1958) et Les Têtes de Pioche (1976-1979) (Ottawa, Institut canadien de recherches sur les femmes, $\mathrm{n}^{\mathrm{O}}$ 5, 1983 ( $2^{\mathrm{e}}$ édition 1988); Hélène Pelletier-Baillargeon, Marie Gérin-Lajoie. De mère en fille, la cause des femmes (Montréal, Boréal Express, 1985). 
Council of Women, et auquel avaient adhéré Marie Gérin-Lajoie, Caroline Béique, Joséphine Marchand-Dandurand et Marie Thibaudeau. Cependant, l'engagement social des femmes à des fins autres que philanthropiques suscitait la réprobation du clergé catholique canadien-français et de la société en général qui estimaient que la place des femmes était dans leur foyer et ce, malgré l'ouverture créée par la publication de l'encyclique Rerum Novarum de Léon XIII en 1891. En proclamant le droit à l'association pour tous, le pape permettait, en effet, aux femmes catholiques de se regrouper afin de diffuser la doctrine sociale de l'Église. Marie Gérin-Lajoie a rapidement saisi les possibilités offertes par cette encyclique, ainsi que le montre cette note de sa fille:

Quand parut dans notre milieu montréalais, l'encyclique Rerum Novarum éditée par les R.P. Jésuites et qu'elle fut magistralement commentée par le R.P. Archambault à la salle du Gésu, ma mère était dans l'auditoire [...]. Ma mère exultait de joie, au retour elle m'expliqua pourquoi: «Ses espoirs d'une action sociale catholique pouvaient donc se réaliser.» Dès lors, plus que jamais, elle ne voulait pas se contenter de rêver l'avenir; l'heure était venue de travailler à le concrétiser ${ }^{16}$.

En 1902, un premier pas fut franchi avec la création, par la Société Saint-Jean-Baptiste (SSJB), d'un comité de dames patronnesses. Ainsi regroupées, les femmes du comité réussirent à s'affranchir légalement de la tutelle de la SSJB et àcréer, en 1907, la Fédération nationale Saint-JeanBaptiste. Sous la présidence de Caroline Béique, la nouvelle fédération «appelait les femmes dans ses rangs non pas confusément mais par classes définies, afin de mettre en lumière les besoins propres à chacune d'elles et d'améliorer les conditions de vie de toutes ${ }^{17} \gg$. Marie Gérin-Lajoie, succédant à Caroline Béique en 1913, occupa le poste de présidente de la FNSJB durant vingt-cinq ans et en marqua profondément l'idéologie.

La FNSJB, au moment de sa fondation, s'était fixé des objectifs d'action précis. Premièrement, elle voulait créer une «organisation raisonnée et systématique des forces féminines de ce pays en vue de déterminer dans la vie de la femme et dans toutes les manifestations de son activité, un progrès continu et une véritable ascension sociale de notre sexe ${ }^{18}$ 》. L'amélioration des conditions de vie des femmes et la reconnaissance de

16. Cette citation est tirée de Hélène Pelletier-Baillargeon, Marie Gérin-Lajoie..., 92.

17. Marie Gérin-Lajoie, «Étude présentée aux fêtes du $25^{\mathrm{e}}$ anniversaire. Le caractère de la Fédération, le milieu où elle a pris naissance, sa constitution, ses œuvres», BP, 21,1 (février 1933): 1.

18. Marie Gérin-Lajoie, «Allocution de la présidente de la Fédération à l'Assemblée générale du 30 janvier 1916», BP, 4,1 (mars 1916): 4. 
leur rôle social se trouvaient donc au cour de ses aspirations. La restauration sociale sur des bases chrétiennes par le recours à la charité et à l'éducation - autrement dit l'action sociale catholique — constituait l'autre objectif majeur des fondatrices. Selon la conception du monde entretenue par les membres de la FNSJB, ces deux objectifs étaient tout à fait complémentaires, car pour elles, l'étanche séparation des mondes privé et public ne correspondait pas à la réalité. Marie Gérin-Lajoie exprime bien cette position en disant: «Hélas! nous ne le savons que trop: le foyer bien clos, c'est une légende sans réalité: tous les foyers ont des fenêtres sur le dehors et quand l'air du dehors est délétère, ceux qui sont dans la maison peuvent en souffrir, ils en peuvent mourir ${ }^{19}$.» Bref, la FNSJB désirait œuvrer au bien-être des femmes, mais ne pouvait le faire qu'en s'engageant sur le plan de la restauration sociale. En effet, si les mondes public et privé interagissaient, les femmes, pour espérer améliorer leurs conditions de vie, ne devaient pas se terrer dans leur foyer, mais plutôt étendre leur champ d'action et considérer la société comme une famille élargie dans laquelle elles pouvaient jouer le rôle maternel qu'elles remplissaient déjà auprès de leur mari et de leurs enfants.

Au milieu des années 1970, Yolande Pinard présentait un mémoire de maîtrise, Le féminisme à Montréal au commencement du $X X^{e}$ siècle, 1893-1920 20 , dans lequel la FNSJB partageait la vedette avec le Montreal Local Council of Women (MLCW) ${ }^{21}$. S'insérant à la fois dans le courant d'études marxistes et dans celui du féminisme égalitaire qui prévalaient à l'époque, Pinard en venait à défendre l'hypothèse selon laquelle «le caractère bourgeois du mouvement féministe montréalais et le profond attachement de celui-ci à l'idéologie de la famille ne pouvait qu'engendrer l'échec partiel de plusieurs de ses revendications ${ }^{22} \gg$. Constat d'échec partiel que je remets aujourd'hui en question par le recours au nouveau concept de maternalisme qui établit que les femmes du début du siècle ont pu utiliser la maternité dans la définition de leur idéologie et dans leurs revendications. En plus d'établir les grandes lignes de l'histoire organisationnelle, Pinard s'est elle aussi intéressée à l'idéologie de la FNSJB ${ }^{23}$. Elle avançait en 1976 que la Fédération a tenté, parfois maladroitement, un amalgame de trois types de féminisme importés de France et du

19. «Entre nous: L'œuvre de La Bonne Parole», BP, 10,5 (mai 1922): 3.

20. Yolande Pinard, Le féminisme à Montréal au commencement du XXe siècle, 1893-1920, mémoire de maîtrise (histoire), Université du Québec à Montréal, 1976.

21. Le MLCW était la section locale du National Council of Women, une organisation féminine pancanadienne, regroupant une majorité de femmes anglophones et protestantes.

22. Yolande Pinard, Le féminisme à Montréal..., 3.

23. Ibid., 53-58. 
monde anglo-protestant: le féminisme chrétien, le féminisme social et le féminisme de revendication juridico-politique. Le féminisme chrétien, élaboré en France à la suite de la publication de Rerum Novarum, permettait à la FNSJB de concilier les autres formes de féminisme, originaires en grande partie du monde anglo-saxon et protestant, avec la foi catholique de ses membres, tout en se ménageant de rares appuis dans le clergé canadien-français. Les membres de la FNSJB se réclamaient ouvertement du féminisme chrétien et présentaient généralement celui-ci d'une façon assez conservatrice en l'associant directement à l'action sociale catholique. Sœur Marie Gérin-Lajoie, fille de Marie Gérin-Lajoie présidente de la FNSJB, exprime clairement la différence entre le féminisme libéral, ou mauvais féminisme, et le féminisme chrétien: «Au féminisme libéral qui prône le droit au divorce, la limitation des naissances, l'organisation individualiste de la vie et la licence sous tous ses formes, elle [l'Église] oppose le féminisme chrétien, qui veut obtenir pour la femme certains droits, mais en vue de l'accomplissement intégral de ses devoirs ${ }^{24} . »$

De son propre aveu, la Fédération adhérait au féminisme chrétien. Yolande Pinard a relevé cette influence dans l'orientation idéologique de la FNSJB, tout comme elle a décelé l'importance du féminisme social et du féminisme de revendication juridico-politique. Le féminisme social était un courant étroitement lié au réformisme et valorisait un engagement laïque et autonome pour les femmes, principalement dans les organisations de charité. Les œuvres philanthropiques auxquelles participaient les bourgeoises leur avaient donné l'occasion de saisir la détresse de «leurs sœurs» moins fortunées. Le féminisme social «mettait l'emphase [sic] sur les réformes sociales jugées indispensables plutôt que sur la conquête de droits égaux ${ }^{25}$ ». Le féminisme de revendication juridico-politique - troisième type de féminisme mentionné par Pinard — découlait en partie du féminisme social. En effet, les bourgeoises engagées dans les réformes ont pris conscience des limites des conditions juridiques et politiques propres à leur sexe. Confrontées à l'inégalité de leurs droits, ces femmes ont voulu réparer cette injustice et participer activement au bien-être de la société. Ces deux dernières formes de féminisme se complétaient, les droits juridiques et politiques étant nécessaires à l'accomplissement des réformes entreprises. Au-delà de toutes ces formes de féminisme, Pinard admet que les premières féministes du Québec,

24. Sœur Marie Gérin-Lajoie, «Le retour de la mère au foyer», École sociale populaire, 227 (décembre 1932): 2.

25. Ibid., 16. 
tant anglophones que francophones, étaient unanimes à reconnaître la primauté de «l'idéologie de la femme au foyer et de la famille ${ }^{26}$ ». Mais cette reconnaissance n'est pas exploitée à sa juste mesure dans l'analyse qu'elle propose de l'idéologie de la FNSJB.

La Fédération nationale Saint-Jean-Baptiste était avant tout une organisation vouée à l'action. L'idéologie et l'argumentation se rejoignaient donc dans la défense de dossiers précis, la lutte pour l'obtention du droit de vote constitue un exemple intéressant à ce sujet. Je reprendrai plus loin ce dossier très représentatif des positions générales de la Fédération pour illustrer l'analyse qui suit.

\section{L'MAGE DE LA FEMME ET SES FONCTIONS SOCIALES}

À la base de toutes leurs positions idéologiques et de leurs revendications, les membres de la FNSJB entretenaient une vision commune de «la femme». Celle-ci ne détonnait pas du discours général à ce sujet puisqu'il existait au Québec francophone, comme un peu partout ailleurs en Occident et particulièrement dans les pays influencés par l'idéologie victorienne à la fin du $X I X^{e}$ siècle et au début du $X X^{e}$, un assez large consensus à propos de ce que devait être «une femme». Une image idéalisée des femmes, ou plutôt de $\left\langle l a\right.$ femme $\left.{ }^{27}\right\rangle$, circulait tant dans la presse catholique, que politique ou féminine:

La femme, d'après l'idéal des siècles chrétiens, est une ouvre de choix, le chef-d'ourve des mains divines qui, en créant des sours, des mères, des épouses, ont sculpté dans le marbre humain, avec un art infini, les physionomies les plus douces, les vies les plus humbles et les plus dévouées ${ }^{28}$.

Cette façon de définir les femmes comme des sours, des épouses et des mères correspond assez bien à l'analyse faite par Simone de Beauvoir qui affirme, dans Le deuxième sexe, que la femme «se détermine et se différencie par rapport à l'homme et non celui-ci par rapport à elle ${ }^{29}{ }^{2}$. Selon cette thèse, l'homme définit la femme par rapport à lui-même, c'est-à-dire

26. Ibid., 13 .

27. L'utilisation du terme «la femme» était généralisée à cette époque, traduisant une conception unique des femmes. Aujourd'hui, ce terme est remplacé par le pluriel, «les femmes», qui témoigne de la diversité reconnue des situations vécues par les femmes et de leur identité subjective. J'utiliserai donc le terme singulier, la femme, lorsque je ferai référence aux sources, alors que pour l'analyse j'emploierai le terme pluriel, les femmes.

28. $\mathrm{M}^{\mathrm{gr}}$ L.-A. Paquet, «Le féminisme», Études et appréciations. Nouveaux mélanges canadiens (Québec, Imprimerie franciscaine missionnaire, 1919), 9.

29. Simone de Beauvoir, Le Deuxième sexe (Paris, Gallimard Folio, 1976), 1: 15. Première édition, 1949. 
en la posant comme l'autre, tandis que la femme se définit comme l'autre par rapport à l'homme. Cet autre prend alors une silhouette relativement homogène dans la société, autant parmi les femmes que parmi les hommes. La notion de la différence s'impose ainsi à la base de la définition des sexes et la nature en est un critère fondamental. Par son journal mensuel, La Bonne parole, la FNSJB participait à ce discours général sur les femmes. On y apprend, sans grande surprise, que les femmes étaient dans l'absolu douces, humbles, charitables, attentionnées, intuitives, tendres et compatissantes. Toutefois, leur principale qualité morale, celle qui constituait la base du modèle maternel idéal, était le dévouement, relevant luimême dun profond sens du devoir. Georgette LeMbyne, rédactrice à $L a$ Bonne parole, avançait même que ce dévouement représentait le fondement des ouvres de la FNSJB puisque, selon elle, «le dévouement nous a faites ce que nous sommes ${ }^{30}$ 》.

Le modèle maternel par excellence demeurait celui de la Vierge Marie, à qui la FNSJB demandait dans sa prière officielle: «Rendez-nous humbles, charitables, douces, zélées comme vous ${ }^{31} . \gg$ En implorant l'intervention de la Vierge pour les aider à lui ressembler, on comprend que les membres de la FNSJB étaient conscientes qu'il existait une marge entre le modèle parfait de la Vierge et la réalité. Cette image de la femme, bien qu'elle fût un idéal, était partagée par les membres de la FNSJB - fort probablement favorables à une certaine émancipation des femmes - et par les plus farouches adversaires du féminisme comme Henri Bourassa. Dans un texte qui remonte à quelques années déjà, Susan Mann-Trofimenkoff défendait l'hypothèse voulant que derrière l'attachement de Bourassa à un idéal pur, doux et émotif de la femme, se cachait l'image complémentaire qu'il avait de l'homme, violent, raisonné et autoritaire ${ }^{32}$. Cette idée de la complémentarité des sexes faisait, à quelques voix près, l'unanimité. Néanmoins, il apparaît que pour les membres de la FNSJB, si complémentarité il y avait, celle-ci n'impliquait pas nécessairement une inégalité de droits.

À la complémentarité de nature des hommes et des femmes correspondait une complémentarité des rôles dans la famille et dans l'ensemble de la société. Il appert ainsi qu'une certaine conception fonctionnaliste était mise de l'avant pour expliquer la division traditionnelle des tâches entre les hommes et les femmes: la nature déterminait la fonction. En fait,

30. Georgette LeMoyne, «Entre nous. Souhaits», BP, 12,1 (janvier 1924): 3.

31. Georgette LeMoyne, «Entre nous», BP, 9,12 (décembre 1921): 3.

32. Susan Mann-Trofimenkoff, «Henri Bourassa and the Woman Question», Revue d'études canadiennes, 10,4 (1975): 3-11. 
comme l'affirmait Bourassa: «la différence des sexes entraîne la différence des fonctions sexuelles, et la différence des fonctions sexuelles crée la différence des fonctions sociales ${ }^{33} . \gg$ Or la possibilité d'être mère constituait une des principales caractéristiques naturelles des femmes. Il n'est donc pas étonnant que la fonction sociale première des femmes fût la maternité et que toutes les autres fonctions reconnues aux femmes y étaient liées. La vie des femmes était doublement fondée sur la maternité: d'une part, le «besoin de maternité» déterminait leur vie, qu'elles soient mères ou non, en orientant leurs choix et leurs actions; d'autre part, lorsqu'elles étaient mères, ce devoir avait priorité sur tout le reste. LaFNSJB ne percevait toutefois pas la maternité comme une fonction limitative, mais plutôt comme une ouverture vers d'autres tâches sociales. Pourquoi définissait-on la femme par la matemité? Quels éléments étaient impliqués dans cette définition de la fonction maternelle des femmes?

Le recours à la religion constituait une base commune d'explication pour un grand nombre de sujets abordés dans la société canadienne-française de cette époque. Les questions se rapportant aux femmes, ainsi qu'à leur rôle maternel, n'échappaient pas à cette règle et les arguments religieux, utilisés par toutes les parties concernées, n'étaient généralement pas contestés. MFr Paquet explique que «Dieu l'a [la femme] créée pour des fonctions sans lesquelles la famille ne pourrait vivre, ni l'humanité se perpétue ${ }^{34} \gg$. Par ailleurs, la religion catholique endossait la thèse de la complémentarité des hommes et des femmes et en valorisait la concrétisation dans le mariage. La véritable fonction maternelle des femmes ne pouvait se réaliser que dans le cadre conjugal, le seul reconnu comme propice à la procréation. Mais toutes n'étaient pas appelées à se marier et l'Église reconnaissait cette situation en confiant «au plus grand nombre de ses religieuses des tâches précisément maternelles comme sont l'éducation des enfants, le soin des malades, le secours aux pauvres ${ }^{35}$ ».

La plupart du temps, la fonction maternelle des femmes était présentée en rapport avec le milieu qui se prêtait le mieux à son exercice: la famille. Sy conjuguaient un certain nombre de fonctions complémentaires: épouse, éducatrice et ménagère. En effet, si la femme, par son rôle dépouse, devait veiller — tout en y étant soumise —à la bonne conduite de son mari, elle devait surtout, en raison de son rôle de mère, éduquer ses

33. Cette citation est tirée de Diane Lamoureux, «Une opposition farouche au suffrage féminin, 1913-1940», dans Robert Lahaise, dir., Le Devoir, reflet du Québec au $20^{e}$ siècle (LaSalle, Éditions Hurtubise, 1994), 333.

34. $\mathrm{M}^{\mathrm{gr}}$ Paquet, «Le féminisme», 33 .

35. Sœur Marie Gérin-Lajoie, «Le retour de la mère au foyer», 3. 
enfants selon les principes de la morale catholique, car «la femme a une mission moralisatrice, et c'est logique puisque par elle se perpétue l'humanité. Elle est l'éducatrice-née du genre humain ${ }^{36} 7$. En outre, les responsabilités matérielles et physiques des mères n'étaient pas à négliger. Au début du siècle, ces dernières obligations prirent de plus en plus dimportance dans le contexte de la lutte contre la mortalité infantile amorcée peu avant la Première Guerre mondiale grâce aux efforts des réformistes, parmi lesquels la FNSJB occupait une place importante à Montréal. Est-il besoin de rappeler que Montréal détenait, au début du siècle, un record peu enviable de mortalité infantile ${ }^{37}$ ? Bref, les mères étaient responsables de tous les aspects du développement de leurs enfants, tant au point de vue moral, que religieux ou physique.

Selon la FNSJB, si c'est dabord à ses enfants et à sa famille qu'une mère se devait, elle était aussi tenue de concevoir son rôle dans un plus large horizon: «Être mère c'est avoir conscience que par son rôle d'éducatrice naturelle, on va préparer l'homme de demain, on va donner à l'état [sic] le facteur nécessaire à sa vie économique et sociale ${ }^{38} . \gg$ La famille était considérée comme la cellule de base de la société et cette perception de la vie sociale a rendu possible l'élargissement des frontières familiales. Dans le cadre du congrès de l'Union internationale des ligues catholiques féminines (UILCF) de 1925, Marie Gérin-Lajoie nous offre sa conception de la famille: «Non pas la famille au sens étroit du mot, avec ses portes closes et sans vue sur le monde extérieur, comme s'il était possible de se soustraire à la loi de la solidarité; mais la famille rayonnante, génératrice de vie et d'énergies sociales, où s'élabore la pensée qui demain prendra une forme concrète dans les lois et l'administration publique ${ }^{39} . \gg$ Dans cette perspective, on comprend que, de l'avis de la FNSJB, les femmes, si elles jouaient un rôle matemel important dans la famille, devaient occuper une place correspondante dans la société:

Certes, le foyer est le centre naturel de l'action de la femme; nulle auréole ne lui convient mieux que celle d'épouse et de mère; c'est

36. A. Goutay, Fédération des femmes belges, «La mission de la femme», BP, 23,9 (septembre 1935): 13 .

37. Denyse Baillargeon retrace quelques statistiques à ce sujet. En 1910, le taux de mortalité infantile pour la ville de Montréal était de 224 décès pour 1000 naissances chez les Canadiens français. Statistiques tirées de Denyse Baillargeon, «Fréquenter les Gouttes de lait. L'expérience des mères montréalaises, 1910-1965», Revue d'histoire de l'Amérique française, 50,1 (été 1996): 30. 10

38. Pauline Fréchette-Handfield, «L'art d'être une bonne mère», BP, 23,9 (septembre 1935):

39. Marie Gérin-Lajoie, «Chronique internationale. Le Congrès de l'Union Internationale des Ligues Catholiques féminines», BP, 13,12 (décembre 1925): 4. 
l'ouvre de la création par excellence. Cependant, au-dessus de ce foyer, de la famille, il y a une autre grande famille qu'on appelle la Société, qui réclame les mêmes attentions. Or, dans cette société doit régner la même harmonie que dans la famille qui en est la base. Cestà-dire que la collaboration de la femme y est aussi nécessaire ${ }^{40}$.

L'élargissement du rôle familial et matemel des femmes constitue un des fondements du matemalisme. De cet élargissement sont nées des dissensions importantes entre la FNSJB et d'autres intervenants sociaux comme le clergé. Car si tous semblaient s'entendre sur le modèle idéal féminin, des divergences émergeaient à propos de l'adaptabilité de cette «nature féminine» à la société en évolution. La FNSJB et ses alliés valorisaient cette adaptabilité afin que les femmes puissent exercer leur influence morale dans la société, de la même manière qu'elles le faisaient dans la famille. En revanche, à la fois ceux qui étaient réfractaires au changement en général et de nombreux autres plus progressistes voyaient comme une menace les initiatives de ces femmes.

Pour la Fédération, la vie nationale a constitué un des théâtres daction privilégiés pour réaliser l'élargissement de leur fonction matemelle. Puisque la nation, à l'image de la société ${ }^{41}$, reposait sur l'ensemble des familles qui la composaient et que, pour la FNSJB, les femmes constituaient les fondements de la famille, elles étaient par conséquent à la base de la nation. Marie Gérin-Lajoie résume ici cette pensée élaborée alors qu'elle était présidente de la FNSJB: «Mais la nation n'est pas un tout individuel sur lequel on puisse agir. Elle dépend directement et tire toute sa valeur des familles qui la composent. Cest un devoir éminemment patriotique d'assurer la stabilité et la dignité du foyer ${ }^{42}$.» Les femmes pouvaient donc exercer une fonction de patriotes dans la famille et, par conséquent, influer sur la société. Dans la mesure où la famille demeurait la scène privilégiée du patriotisme féminin, les oppositions se sont faites discrètes, entre autres parce qu'une partie de l'identité nationale canadienne-française reposait sur la famille nombreuse, illustration de la vitalité de la «race». Le Premier ministre Louis-Alexandre Taschereau, lors d'une conférence donnée au congrès annuel de la FNSJB en 1923, s'est attardé à définir ce lien entre les femmes, les familles nombreuses et le sentiment patriotique: «Cest à la femme canadienne-française que nous devons ce que nous avons de

40. «Les Cercles des Fermières», BP, 12,11 (novembre 1924): 1.

41. Nous avons constaté une certaine confusion dans l'utilisation des termes nation et société dans La Bonne parole, tous deux paraissant être interchangeables.

42. Marie Gérin-Lajoie, «Entre nous», BP, 3,5 (juillet 1915): 1; le souligné est de M. GérinLajoie. 
meilleur et ce que le voisin nous envie le plus: la famille nombreuse, robuste, attachée au sol, fidèle à sa langue, à sa foi et conservant fièrement le culte aux aïeux, l'amour de la vieille mère patrie ${ }^{43}$.»

La Première Guerre mondiale est venue modifier légèrement la définition du patriotisme féminin que les membres de la FNSJB avaient jusque-là partagée avec l'ensemble de la société. Les femmes ont été appelées à s'engager directement dans l'effort de guerre, comme en témoigne cet éditorial de la rédactrice en chef de La Bonne parole, Madeleine Huguenin, paru en 1918:

La femme est appelée dans toutes les nations à prendre sa part plus large de responsabilités, à sauvegarder le présent, à préparer l'avenir, à assurer en un mot la vie des peuples, non seulement dans la mission sacrée qui est sa fonction naturelle, mais encore dans l'accomplissement de besognes qui ont été jusqu'ici épargnées à ce que l'on voulait bien appeler sa faiblesse ${ }^{44}$.

Dans la suite de cet éditorial, Madeleine Huguenin expliquait que ces nouvelles responsabilités allaient se résorber d'elles-mêmes après la guerre, car les femmes n'aspiraient qu'à retourner dans leurs foyers. Conscientes de leurs capacités, elles allaient cependant être en mesure de se délivrer des asservissements qui avaient été leurs par le passé. Bref, l'appel à leur sentiment patriotique pendant la Première Guerre mondiale a permis aux femmes de prendre conscience de l'ampleur de leur rôle dans la nation. Il n'en demeurait pas moins que la meilleure façon - mais non plus la seule - pour les femmes d'être patriotes, c'était encore d'être mères.

Conscientes du rôle accru qu'elles pouvaient jouer dans la société, mais confrontées aux limites et aux défauts du monde dirigé par les hommes, ces femmes se sont interrogées sur les manières de concilier modernité et catholicisme, de même que sur les moyens à prendre pour panser les plaies sociales mises à vif par la guerre et la crise. Elles ont voulu contribuer à la régénération sociale en faisant profiter la société de leurs qualités féminines ainsi qu'en appliquant les grands principes religieux établis par la doctrine sociale catholique et par Rerum Novarum. Elles se sont donc présentées comme des régénératrices sociales, encouragées dans cette voie par le discours religieux. Maria Auclair, membre active de la FNSJB, en témoigne dans une conférence: «On demande toujours davan-

43. L.-A. Taschereau, «De l'influence de la femme sur nos destinées nationales», $B P, 9,5$ (mai 1923): 7.

44. Madeleine Huguenin, «Entre nous», $B P, 5,11$ (janvier 1918): 1. 
tage à notre dévouement, et on compte plus que jamais sur la collaboration de la femme, pour apaiser les esprits énervés par les souffrances sans fin que la guerre a suscitées: cherté de la vie, chômage, haines des classes, instabilité du travail ${ }^{45}$.» Mais comment les femmes pouvaient-elles concrétiser cette fonction de régénération sociale? Tout comme dans le cas du patriotisme féminin, celle-ci s'exerçait en premier lieu dans la famille, car le premier devoir des femmes était «celui de reconstruire l'humanité par la "revanche des berceaux"; celui de consolider sur de saines bases de moralité, d'économie et d'éducation la famille, ébranlée par ces luttes fratricides ${ }^{46}$ 》. Mais, élargissant plus ouvertement les cadres familiaux, les membres de laFSNJB se sont engagées dans divers dossiers d'ordre public comme la lutte antialcoolique, la prostitution, la 〈traite des blanches» et la moralité vestimentaire des femmes ${ }^{47}$. Justifiée, entre autres, par ce qu'elles croyaient être le sens moral supérieur des femmes, leur intervention visait à protéger la moralité de la société canadienne-française et ainsi à compléter le rôle social des organisations religieuses et de l'État:

Nous avons vu enfin, comment les gouvernements, les institutions, qui ont pour mission de réagir contre ces maux, de protéger le citoyen, sont parfois insuffisants, n'ont pas toujours «l'âme» voulue, pour combattre le mal là où il se trouve. Devant ces pénibles constatations, nous ne pouvons plus dire que la femme n'a aucun devoir à accomplir en dehors du cercle intime de sa famille ${ }^{48}$.

Bref, une vision plutôt fonctionnaliste prévalait à propos des femmes dans le Québec de la première moitié du $X X^{e}$ siècle. La femme, telle que définie par la société canadienne-française et par la FNSJB, était socialement perçue comme le fondement moral de la famille, unité de base de la société. Mère, éducatrice, épouse, ménagère, patriote, régénératrice sociale et chrétienne, voilà les principales fonctions, et même les missions, des Canadiennes françaises. Chacune pouvait se réaliser doublement. En effet, si la famille demeurait le cadre le plus acceptable des réalisations féminines, les membres de la FNSJB furent, selon toute vraisemblance, parmi les premières à soutenir que la société dans son ensemble ne pouvait se passer de l'influence bienfaisante des femmes. Cet élargissement du cadre d'action et d'influence des femmes esquissé par la FNSJB ne chan-

45. Archives nationales du Québec à Montréal [ANQM], Fonds FNSJB, 06, MP/120, bobine 6826, manuscrit de conférence donnée par Maria Auclair, c1919, 1.

46. Georgette LeMoyne, «Entre nous», BP, 6,9 (novembre 1918): 1.

47. Le dossier de la moralité vestimentaire fut en fait la lutte première d'une organisation affiliée à la FNSJB, la Ligue des femmes chrétiennes.

48. Georgette LeMoyne, «Des devoirs et de l'éducation civiques de la femme», $B P, 16,1$ (janvier 1928): 5 . 
geait toutefois pas la nature de leurs fonctions, qui reposaient toujours sur leur «mission» maternelle première.

En sachant que les membres de la Fédération acceptaient leur «nature féminine» et la mission matemelle qui en découlait, on comprend la pertinence de vérifier jusqu'à quel point cette position déterminait leur orientation idéologique générale. Il faut bien comprendre que les membres de la FNSJB ne se définissaient pas comme des maternalistes. En parlant d'elles-mêmes, elles employaient plutôt le terme féministe. Par ailleurs, toutes les nuances employées et les efforts mis à dénoncer le «mauvais» féminisme me portent à croire que des réserves importantes existaient dans leur esprit au sujet du radicalisme souvent associé au féminisme. Tout cela mamène à suggérer le maternalisme comme catégorie d'analyse capable déclairer les décisions de la FNSJB. Il ressort effectivement de l'ensemble de la production écrite de la Fédération que la femme ne peut nier sa nature maternelle, différence première entre les hommes et les femmes: «On a beau vouloir dénaturer la femme et en faire un guerrier, la faire concourir aux ourres de mort, elle revient fatalement à sa vocation providentielle dans le monde [...] ainsi la femme, dès qu'elle est livrée à ellemême donne cours à ses élans maternels, assiste ceux qui souffrent et qu'on oppresse, et voue toute son âme aux ourres de vie ${ }^{49}$.»

\section{LE MATERNALISME DE LA FNSJB L'EXEMPLE DU DROIT DE VOTE}

La Fédération était, d'abord et avant tout, une organisation militante, dans laquelle l'idéologie n'avait de sens que dans le développement d'une argumentation destinée à appuyer certains dossiers tels le changement du code civil en faveur des femmes mariées ou l'accès à l'éducation pour les femmes. La lutte pour l'obtention du suffrage féminin constitue un exemple significatif de ce développement argumentatif. Il est d'autant plus intéressant, dans le cadre d'un article comme celui-ci, qu'il s'agit d'un dossier assez bien connu et déjà abondamment documentés ${ }^{5}$. Le choix de ce dos-

49. Marie Gérin-Lajoie, «Entre nous», BP, 6,1 (mars 1918): 1.

50. Pour avoir une idée des acteurs et des événements qui ont marqué ce débat: Maryse Darsigny, Du Comité provincial du suffrage féminin à la Ligue des droits de la femme (1922-1940): le second souffle du mouvement féministe au Québec de la première moitié $d u X X^{e}$ siècle, mémoire de maîtrise (histoire), Université du Québec à Montréal, 1994; Yolande Pinard, Le féminisme à Montréal...; Luigi Trifiro, «Une intervention à Rome dans la lutte pour le suffrage féminin au Québec, 1922», Revue d'histoire de l'Amérique française, 32,1 (juin 1978): 3-18; Collectif Clio, L'histoire des femmes au Québec (Montréal, Le Jour, 1992). Cette liste est loin d'être exhaustive, mais elle permet de comprendre les grandes étapes du dossier, de ses débuts à la fin du XIX ${ }^{\mathrm{e}}$ siècle, jusqu'à l'obtention du suffrage féminin au Québec, en 1940. 
sier n'est donc pas gratuit, tout le débat entourant l'accession des femmes aux franchises électorales, em raison de son caractère public, ayant souvent été décrit comme l'élément représentatif de l'ensemble du mouvement féministe: qui était suffragiste était féministe. Aujourd'hui, l'équation n'est plus automatique. On reconnaît que l'appui au suffrage n'était pas toujours synonyme d'un vote pour «l'émancipation de la femme». De la même façon, l'opposition au vote des femmes, loin dêtre toujours l'apanage des conservatrices, peut avoir été la position d'anarchistes comme Emma Goldman aux États-Unis, qui ne croyaient pas que les femmes, pas plus que les hommes d'ailleurs, puissent profiter de la démocratie telle qu'elle existait. Le spectre des positions idéologiques et politiques des mouvements féminins se traduisait donc dans les différents arguments engagés dans le débat sur le droit de vote des femmes.

Avant d'aller plus loin dans l'examen des positions idéologiques et des arguments de la Fédération, il est nécessaire de rappeler brièvement les grandes étapes qui ont mené à l'accession des femmes québécoises au suffrage. Les revendications pour le suffrage féminin s'inscrivent dans un mouvement occidental, que l'on peut lier à la vague de réformes sociales qui a émergé en réaction à l'industrialisation. Les femmes ont participé à ces réformes, qu'elles ont même souvent préparées. Ce fut pour elles l'occasion d'une révélation de leur condition sociale, juridiqueet politique. Réformistes, elles voulaient changer les choses, mais n'en avaient pas les moyens. Elles ont donc revendiqué, entre autres, le droit de participer directement à la vie politique en utilisant le droit de vote, puisque la majorité des pays occidentaux fonctionnaient sous un régime démocratique.

Mais les hommes en place n'ont pas toujours vu d'un très bon oeil cette intrusion féminine dans le monde politique masculin. Des débats se sont amorcés un peu partout, mais sans succès immédiats pour les femmes ${ }^{51}$. Il faudra attendre un événement perturbateur majeur, la Première Guerre mondiale, pour que les portes commencent à s'ouvrir pour elles. Les femmes britanniques ont obtenu le suffrage en 1919 et les Américaines en 1920. Absente de la lutte qui mena, en 1917, au suffrage des femmes au Canada, la FNSJB s'imposa pourtant comme chef de file du mouvement qui prit forme la même année au niveau provincial ${ }^{52}$. Un débat de longue

51. Sauf dans certains pays comme la Nouvelle-Zélande et l'Australie où le suffrage a été accordé aux femmes dès les années 1890 .

52. Le gouvernement Borden accorda un droit de vote limité aux femmes en 1917; seules les parentes de soldats et les infirmières engagées dans l'armée pouvaient s'en prévaloir. À la suite de pressions exercées par des groupes de femmes - et la FNSJB entra en scène à ce moment - le gouvernement Borden généralisa le suffrage féminin l'année suivante, à toutes les femmes âgées de 21 ans et plus. 
haleine s'est alors installé au Québec dans lequel la Fédération a joué un rôle plus ou moins dominant selon les étapes. En 1922, la Fédération et le MLCW décidèrent d'unir leurs forces en créant un comité indépendant, le Comité provincial du suffrage féminin (CPSF), dont l'unique objectif était d'obtenir le droit de vote pour les Québécoises.

Toujours en 1922, une virulente offensive antisuffragiste fut menée tant dans la presse que parmi le clergé et le monde politique. Certaines organisations de femmes, dont les Cercles des fermières, s'y sont jointes pour contrer les efforts suffragistes qui s'organisaient dans la province, mais surtout à Montréal. Marie Gérin-Lajoie déploya, en vain, ses énergies pour faire tomber les résistances, celles des membres du clergé en particurlier. Ele se rendit même à Rome pour y demander un avis officiel ${ }^{53}$. L'avis solliciténe condamnait pas la participation politique des femmes, mais exigeait «que toute nouvelle initiative, sur le terrain du suffrage féminin, soit soumise d'avance dans chaque pays à l'approbation de l'Épiscopat ${ }^{54} \gg$. Or l'Épiscopat du Québec était réfractaire à cette mesure et n'y voyait pas une nécessité; contrairement à certains pays européens, le catholicisme était peu menacé par le communisme dans la province, et il n'avait pas besoin de l'appui politique, présumé conservateur, des femmes. Après cette rebuffade, Marie Gérin-Lajoie démissionna du Comité provincial du suffrage féminin, qui suspendit alors ses activités jusqu'à ce qu'il se scinde en 1927. Thérèse Casgrain, qui avait accédé à la présidence du Comité, en relança alors les activités ${ }^{55}$. La même année, Idola Saint-Jean quitta son poste de secrétaire du CPSF pour fonder l'Alliance canadienne pour le vote des femmes. Sengagèrent alors des «pèlerinages» annuels à Québec dans le but d'appuyer, en tribune, le député qui avait accepté de déposer un projet de loi visant à octroyer le vote aux femmes. Il faut dire que le monde politique —et les premiers ministres Taschereau et Duplessis —était généralement hostile aux revendications politiques des femmes. Les appuis de ce milieu se firent donc toujours de façon individuelle et jamais la ligne de parti ne fut imposée aux députés pour appuyer ceux des leurs — majoritairement libéraux — qui déposèrent les projets de loi entre 1927 et $1940^{56}$. Cest

53. Cet événement de la campagne suffragiste est décrit par Luigi Trifiro dans son mémoire La crise de 1922 dans la lutte pour le suffrage féminin au Québec, mémoire de maîtrise (histoire), Université de Sherbrooke, 1976.

54. Rapport de l'UILCF, $5^{\mathrm{e}}$ Conseil International, Fonds FNSJB. Cette note est tirée de Luigi Trifiro, «Une intervention à Rome...», 10.

55. Thérèse Casgrain changea le nom du Comité pour celui de Ligue des droits de la femme en 1928, marquant ainsi un élargissement des orientations de l'association.

56. Pour la liste des députés concernés, voir Diane Lamoureux, Citoyennes? Femmes, droit de vote et démocratie (Montréal, Éditions du remue-ménage, 1989), 50. 


\section{finalement en 1940, en partie grâce aux démarches de Thérèse Casgrain, que le droit de vote fut concédé aux femmes par le Premier ministre God- bout.}

De 1917 à 1933, années durant lesquelles la FNSJB a participé, plus ou moins activement selon les années, au débat sous la gouverne de Marie Gérin-Lajoie, les arguments utilisés sont restés les mêmes. Le raisonnement de la Fédération reposait essentiellement sur l'image traditionnelle de la femme, ainsi que sur sa conception novatrice des relations entre les mondes privé et public ${ }^{57}$. Marie Gérin-Lajoie constituait la figure de proue de la FNSJB dans l'élaboration de son discours argumentatif et l'établissement de relations avec les autres organisations ou personnes engagées dans la même lutte.

J'ai souligné plus haut que les membres de la FNSJB n'ont jamais remis en question la «nature féminine». Logiquement, le principal argument qu'elles utilisaient dans leur plaidoyer en faveur du suffrage féminin était celui de la différence entre les hommes et les femmes. Au cours d'une allocution présentée devant le Premier ministre Taschereau en 1922, Marie Gérin-Lajoie défendait ainsi sa position:

Il peut vous paraître inattendu, messieurs, que je me prévale du rôle familial de la femme pour réclamer ses droits politiques, tant d'autres ne les ayant sollicités qu'à cause de la participation que la femme apporte au développement économique d'un pays, [...]. Je pense cependant que la raison qui milite le plus en faveur du suffrage pour la femme est celle qui s'appuie sur le caractère spécial et distinctif de sa fonction sociale, et que c'est plus au nom de la différence que de sa ressemblance avec l'homme qu'elle doit jouir des franchises électorales ${ }^{58}$.

La maternité, différence fondamentale entre les femmes et les hommes, était reconnue pour conférer des connaissances particulières aux femmes en ce qui concerne l'éducation, la santé et l'assistance sociale ${ }^{59}$. Les membres de la FNSJB soutenaient que ces compétences étaient

57. Certains éléments nouveaux se sont tout de même glissés dans le discours de la Fédération à la suite du passage de Thérèse Casgrain dans l'organisation. Pour elle, le droit de vote n'était pas un privilège mais bien un droit fondamental des citoyens et les femmes devaient y avoir droit au même titre que les hommes. On peut consulter deux articles de Casgrain publiés dans La Bonne parole: Thérèse Casgrain, «L'attitude de la femme envers les partis politiques», $B P, 16,1$ (janvier 1928): 8; et «Les revendications féminines», $B P, 18,12$ (décembre 1929): 9-10.

58. ANQM, Fonds FNSJB, 06, MP/120, bobine 6835, Marie Gérin-Lajoie, Le vote féminin et la question familiale. Plaidoyer en faveur du suffrage féminin fait devant les ministres et les députés réunis, à Québec, le 9 février 1922, 7a.

59. Marie Gérin-Lajoie, «Entre nous», BP, 7,3 (mai 1919): 1. 
nécessaires au bon fonctionnement de la société mais que, pour les faire valoir, les femmes devaient obtenir au préalable le droit de vote. Le suffrage féminin devenait ainsi une responsabilité sociale tout à fait conforme à la nature féminine.

Les nombreuses interrogations de Marie Gérin-Lajoie lui avaient également permis de se rendre compte de l'artificialité des frontières qui existaient entre les sphères privée et publique et qui limitaient l'action des femmes à la première. En 1918, dans un éditorial de La Bonne parole, elle remettait en question ces frontières et proposait plutôt une vision globale du monde:

\begin{abstract}
Ainsi conçue, la collaboration de la femme aux questions d'intérêt commun se présente à elle sous un aspect sévère et son activité extérieure est motivée, non par des raisons puériles ou de mesquines rivalités de sexe et des théories déclamatoires sur l'égalité de l'homme et de la femme; mais elle est justifiée par le fait que la fonction sociale de la femme différant par certains côtés de celle de l'homme, elle seule peut savoir dans quel sens elle doit la développer, la perfectionner et introduire dans sa propre vie ce progrès que la vie publique communique à la vie privée $e^{60}$.
\end{abstract}

Il s'agit là d'un aspect fondamental du maternalisme, très présent dans la vision du monde proposée par la FNSJB. Sans ce lien incontournable entre les sphères privée et publique, Marie Gérin-Lajoie, n'aurait pas pu élargir le rôle maternel des femmes à l'ensemble des secteurs sociaux. D'autres membres importants de l'organisation se sont penchées sur l'évolution de la société et ont tiré les mêmes conclusions que leur présidente. Georgette LeMoyne, longtemps secrétaire de la FNSJB, expliquait que «de nos jours, dans nos sociétés modernes, on ne peut même pas toujours dire où finit la vie de famille et où commence la vie sociale, économique, politique, tellement tous les intérêts s'appellent, se touchent, se confondent ${ }^{61}{ }_{\gg}$. On a longtemps pensé que l'ensemble des hommes et des femmes acceptait sans trop de questionnement le cloisonnement social privé/public, accentué par le changement de mode de production issu de l'industrialisation. La méprise était aisée, puisque même les mouvements de revendication féminins modérés, comme la FNSJB, ne remettaient pas vraiment en question la primauté du rôle maternel - et généralement associé à la sphère privée - de la femme. Un son de cloche quelque peu différent se fait toutefois entendre à la lecture des documents produits par

60. Marie Gérin-Lajoie, «Entre nous», BP, 7,7 (octobre 1918): 1. Je souligne.

61. Georgette LeMoyne, «Des devoirs et de l'éducation civiques de la femme», $B P, 16,1$ (janvier 1928): 5. 
la FNSJB. Tout comme la grande majorité des intervenants sociaux, dont le clergé et les politiciens, la Fédération appuyait le partage des rôles sociaux $^{62}$, mais elle en préconisait un élargissement significatif. En d'autres mots, l'acceptation d'une division des rôles sociaux entre les hommes et les femmes n'entraînait pas nécessairement une équation entre les rôles sociaux féminins et la sphère privée. La fonction maternelle et première des femmes s'exerçait, bien entendu, dans la famille, mais aussi dans la société, à la fois directement, notamment par la participation directe des femmes aux décisions politiques, et indirectement, par osmose entre la famille et la société.

La compréhension de la place des femmes à partir de leur rôle social, lui-même basé sur leur maternité potentielle, était partagée autant par la FNSJB que par les opposants au suffrage féminin, mais les deux camps l'employaient différemment selon la position qu'ils défendaient. Cette différence dans l'utilisation reposait justement sur la conception quelque peu divergente qu'ils avaient de la société. Si tous s'entendaient pour dire que les hommes et les femmes étaient complémentaires, les adversaires du féminisme et du suffrage féminin percevaient une frontière entre ces deux mondes que les hommes étaient seuls à pouvoir franchir; ils ne pouvaient accepter que les femmes la traversent, ce qui aurait alors signifié une rivalité entre les hommes et les femmes menant inévitablement à la ruine de la famille et, par conséquent, à celle de la société. On l'a vu, la FNSJB défendait une vision complémentaire des deux mondes. Si les femmes détenaient une place importante dans le monde privé - ce sur quoi tout le monde s'entendait - elles devaient, de l'avis de la Fédération, en avoir une équivalente dans le monde public. C'est dans cette perspective qu'elle réclamait le droit de vote, afin que les femmes puissent assumer leurs responsabilités et leurs fonctions dans une société moderne. Marie Gérin-Lajoie n'a-t-elle pas soutenu que «les questions politiques ont chacune une assise dans la famille ${ }^{63} \gg$ ? La complémentarité des deux mondes ressemble ici à une interaction dans laquelle la participation

62. Andrée Lévesque résume bien le discours dominant sur le sujet: «Pour confirmer la séparation entre la sphère publique et la sphère privée, les grandes qualités maternelles sont invoquées. Leur inscription dans la nature et dans les vues divines se retrouvent dans les enseignements de l'Église et de la médecine. Les penseurs catholiques fondent ce clivage fonctionnel et sur la nature et sur la tradition paulinienne sur la soumission des femmes, reprise par Thomas d'Aquin, pour leur rappeler que leur supériorité morale, loin de les destiner à un rayonnement public, doit s'exercer au foyer et, à la rigueur, dans la philanthropie», La norme et les déviantes. Des femmes au Québec pendant l'entre-deux-guerres (Montréal, Éditions du remue-ménage, 1989), 41.

63. Marie Gérin-Lajoie, «Les femmes et le Sénat», $B P, 8,11$ (novembre 1929): 3. 
démocratique, le droit de vote, pouvait jouer le rôle de courroie de transmission.

Le refus de revendiquer des droits pour eux-mêmes, en motivant toujours ses demandes par la nécessité de se munir de moyens pour permettre aux femmes d'exercer leurs devoirs féminins et maternels, constituait un autre point majeur de la définition idéologique et de l'argumentation de la FNSJB. Cette orientation diffère du féminisme oppositionnel et révolutionnaire tel que défini par Nancy $\mathrm{Cott}^{64}$. Bien sûr, on peut se demander si la position conventionnelle de la FNSJB ne relevait pas d'un certain opportunisme, et si la notion de devoir n'était pas uniquement utilisée dans le but de faire taire ceux qui ne voulaient pas accorder de nouveaux droits aux femmes. Un article de Georgette LeMoyne, publié dans La Bonne parole en 1928, le laisserait supposer:

Les droits de la femme! Voilà de nos sœurs bien plus hardies que nous qui ne traiterons que de ses devoirs civiques, et cela afin de ne pas trop effaroucher certains esprits chagrins, qui s'attristent dès qu'ils entendent parler pour la femme, de justice, de droits, de revendications, mais s'épanouissent, s'il s'agit pour elle au contraire, de devoirs, de souffrances, de renoncements, de responsabilités ${ }^{65}$.

La suite de l'article vient nuancer la position quelque peu cynique de la secrétaire de la FNSJB. Elle y adoptait une position nettement plus favorable au discours majoritaire qui prônait la concession de droits civiques aux femmes en fonction du rôle qu'elles pouvaient jouer dans la société: «On le voit, d'un bout à l'autre du monde, les préoccupations des femmes catholiques restent les mêmes sur cette grave question: considérer la participation de la femme à la vie publique, comme un devoir et un apostolat ${ }^{66} . »$ L'ensemble de la société, hommes et femmes confondus, était baigné dans un discours inspiré à la fois de la religion catholique et de la démocratie libérale, dans laquelle un rôle social est défini pour tous, «rôle auquel doit collaborer chaque élément de la race puisqu'il est fait de gestes de chacun, s'ajustant harmonieusement les uns aux autres et groupant, en un faisceau infrangible, les âmes dont ils relèvent ${ }^{67}$ ».

En plus de concevoir les droits des femmes comme une récompense pour services rendus à la société, Marie Gérin-Lajoie expliquait que les

64. Nancy Cott, «What's in a Name...», 828 .

65. Georgette LeMoyne, «Des devoirs et de l'éducation civiques de la femme», $B P, 16,1$ (janvier 1928): 4.

66. Ibid., 5 .

67. R.P. Archambault, «La Semaine Sociale de Montréal. Sa raison d'être, ses travaux, son esprit», Semaine sociale du Canada (Marianapolis, Edmour Hébert (Censor librorum), 1920), 16. 
femmes devaient prouver à la société, par le bienfait de leurs actions, qu'elles méritaient les droits qu'elles revendiquaient: «Ce qui caractérise le mouvement féministe au Canada, c'est la conscience que la femme prend de ses responsabilités; et avant d'impressionner l'opinion publique par la légitimité de ses revendications, elle s'impose à lui par la force de son action ${ }^{68}$.» La Première Guerre mondiale, en Europe surtout mais aussi au Québec, a donné aux femmes l'occasion de prouver leur valeur et le bien-fondé de leurs demandes: «Le monde devra s'en souvenir: c'est à travers ses œuvres qu'il conviendra désormais d'apprécier et de juger le féminisme, épuré et anobli et, disons-le hautement, après avoir été à la peine, sa place sera à l'honneur ${ }^{69}$.»

Mais quels étaient ces devoirs et ces rôles au nom desquels étaient revendiqués des droits? La rédactrice de La Bonne parole, Yvonne Letellier de Saint-Just, nous l'apprend dans un éditorial de 1932: «Avec les époques, on peut dire que les formes extérieures du rôle familial de la femme ont varié, bien qu'elles se soient toujours ramenées au devoir naturel et primordial de la femme ici-bas: la maternité ${ }^{70} . »$ Elle aussi affirmait que le rôle des femmes ne se limitait plus à ce qu'il a pu être, que le foyer ne pouvait plus être envisagé comme une cellule sociale autonome. En conséquence, la «responsabilité [de la femme] est plus grande, ses obligations se multiplient; niera-t-on qu'elles aient engendré des droits ${ }^{71}$ ?». La primauté de la notion de devoir sur celle de droit ${ }^{72}$ laisse entrevoir un mouvement centré beaucoup plus sur la collectivité que sur l'individu, rapprochant ainsi la FNSJB du courant maternaliste tel que présenté par Molly Ladd-Taylor ${ }^{73}$. Cependant, il est difficile de reprendre les catégories élaborées pour les mouvements américains de maternalisme sentimental et progressiste dans le cas de la FNSJB. En effet, elle allait beaucoup plus loin dans ses revendications que les Cercles des fermières notamment, mais elle ne s'avançait pas à réclamer un salaire familial comme l'ont fait les maternalistes progressistes américaines. Cependant,

68. Archives de l'Institut Notre-Dame-du-Bon-Conseil [AINDBC], Fonds de Marie LacosteGérin-Lajoie, P2, boîte 46, dossier 5, lettre de Marie Gérin-Lajoie à M. Truman (?), Montréal, 18 septembre 1908, 1.

69. Frère Dunan, «Le féminisme et la guerre», $B P, 4,6$ (août 1916): 8.

70. Yvonne Letellier de Saint-Just, «Les droits de la femme sont nés de ses devoirs», $B P, 20,3$ (mars 1932): 1.

71. Ibid., 3 .

72. Cette conception du droit de vote est assez conforme au discours démocratique libéral du $\mathrm{XIX}^{\mathrm{e}}$ siècle, dans lequel on affirmait que le citoyen participe à la vie publique en opérant une distinction entre ses intérêts personnels et ceux de la collectivité à laquelle il appartient. Georges Burdeau, La démocratie (Paris, Éditions du Seuil, 1956), 38.

73. Molly Ladd-Taylor, Mother-Work..., 75. 
il existe, sans aucun doute, une différence fondamentale entre les revendications de la FNSJB, que je qualifie de maternalistes, et celles des féministes de l'époque ${ }^{74}$, ou des Québécoises des années 1960 et 1970, qui revendiquaient des droits en raison de leur citoyenneté et de leur individualité ${ }^{75}$.

La supériorité morale des femmes a également été utilisée par la FNSJB dans sa réclamation du suffrage féminin. Elle soutenait qu'en raison de cette qualité naturelle, la voix des femmes dans les décisions démocratiques pourrait assainir la politique. Dans cette perspective, des articles publiés dans La Bonne parole ont tenté de mettre en lumière l'apport moral positif des femmes à la politique fédérale depuis qu'elles se prononçaient à ce niveau de gouvernement ${ }^{76}$. Les opposants au suffrage féminin partageaient cette perception idéalisée de la supériorité morale des femmes, mais ne l'appliquaient pas nécessairement au monde politique, comme en témoigne Henri Bourassa: «La moralité de la femme, supérieure à celle de l'homme dans la vie privée, quand elle est soutenue par la maternité, n'est ni plus forte ni plus sûre que celle de l'homme en fonction des affaires publiques ${ }^{77}$.» Ils estimaient plutôt, par une logique un peu particulière, que la femme était au-dessus de la politique et qu'elle ne devait pas s'y abaisser si elle voulait conserver cette supériorité morale qui lui conférait une influence profonde sur le fonctionnement social. $\mathrm{M}^{\mathrm{gr}}$ Paquet, qui a écrit un texte nettement en opposition au féminisme et au droit de vote des femmes, exprime cette position en disant: «Moins la femme se commet dans la poussière de la rue et la mêlée des partis, plus sa personne s'impose à la considération publique $^{78}$.»

Dans le dossier particulier du droit de vote des femmes, la FNSJB a dû démontrer que sa position n'allait pas à l'encontre de la doctrine de l'Église. Et ce ne fut pas là une mince tâche parce que les appuis au sein du clergé étaient rares, le père Ceslas Forest de l'Université de Montréal ayant été un des seuls à prendre publiquement position en faveur du suf-

74. Dans le Québec des années 1930, Thérèse Casgrain, avec la Ligue des droits de la femme, et Idola Saint-Jean, avec l'Alliance canadienne pour le vote des femmes, semblent s'être rapprochées de cette position civique en ce qui concerne le droit de vote des femmes.

75. Pour une bonne idée de ce recours à la notion de citoyenneté dans les revendications féministes, on peut consulter Joan Scott, La citoyenne paradoxale. Les féministes françaises et les droits de l'homme (Paris, Albin Michel, 1998).

76. Voir entre autres, Marie Gérin-Lajoie, «Nos droits politiques», BP, 13,10 (octobre 1925): 3.

77. Henri Bourassa, Le Devoir, $1^{\mathrm{er}}$ avril 1918.

78. $\mathrm{M}^{\mathrm{gr}}$ L.-A. Paquet, «Le féminisme», 33. 
frage des femmes ${ }^{79}$. La Fédération n'a d'ailleurs jamais tout à fait réussi à se gagner l'approbation du clergé, et encore en 1940, ce n'est qu'en menaçant le clergé de démissionner en faveur de T.-D. Bouchard - un anticlérical notoire - que le Premier ministre Godbout a pu faire taire les critiques envers son projet de loi et accorder finalement le droit de vote aux Québécoises ${ }^{80}$. De nombreux textes parus dans La Bonne parole ont cherché à démontrer le bien-fondé du suffrage féminin en invoquant le pape. Celui-ci aurait affirmé: «Non seulement je désire que les femmes votent, mais je voudrais les voir voter partout ${ }^{81}$.» Le père Forest affirmait, quant à lui, que le suffrage féminin ne heurtait aucun principe religieux et que le bien commun pouvait même le justifier dans le cas du Québec ${ }^{82}$. De son côté, Marie Gérin-Lajoie était convaincue que les démarches de la FNSJB dans ce domaine controversé n'allaient pas à l'encontre de la doctrine catholique. Elle eut d'ailleurs, jusqu'en 1927 ou 1928, l'appui tacite de $\mathrm{M}^{\mathrm{gr}}$ Gauthier, comme le révèle cet extrait du procès-verbal d'une réunion tenue par le Bureau de direction de la Fédération:

Madame la présidente explique qu'avant d'organiser le Comité du suffrage féminin au provincial, elle a consulté Sa Grandeur Mgr Gauthier [...]. Monseigneur assura de nouveau à Madame GérinLajoie, tout en lui recommandant de chercher le plus possible à apaiser les esprits, que la question ne relève pas du tout de la doctrine catholique, et que sa conscience pouvait être bien tranquille à ce point de vue ${ }^{83}$.

$M^{\text {gr }}$ Gauthier s'est cependant ravisé en 1928 , lorsqu'il a déclaré à l'assemblée annuelle de l'Assistance maternelle - déclaration que $L e$ Devoir s'est empressé de reproduire — que «la femme doit déserter la politique pour se vouer à la charité $e^{84} \gg$. Rien ne nous permet d'expliquer ce changement d'opinion de la part de $\mathrm{M}^{\mathrm{gr}}$ Gauthier. Il était nécessaire, dans une société catholique comme le Québec, de s'assurer de l'orthodoxie dune question aussi importante que le suffrage féminin. Pourtant, malgré toutes les précautions prises par les suffragistes en général, et laFNSJBen particulier, en vue de convaincre le clergé et la société québécoise du bienfondé de leur demande, la majorité des représentants de l'Église ont con-

79. Père Ceslas Forest, «Droit de vote, suffrage féminin et féminisme», La Revue dominicaine, 32 (1926): 385-404.

80. Collectif Clio, Histoire des femmes au Québec..., 364.

81. M.-L. Perrin, «Le suffrage des femmes», BP, 9,11 (novembre 1921): 4.

82. Père Ceslas Forest, «Droit de vote...», 394.

83. ANQM, Fonds FNSJB, 06, MP/120, bobine 6823, procès-verbal du Bureau de direction de la FNSJB, 23 février 1922, 16.

84. Le Devoir, jeudi 5 avril 1928. 
tinué à s'y opposer. Certains, comme MPr Roy de Québec, ont même organisé des campagnes antisuffragistes, en se basant sur la non-conformité supposée du suffrage féminin à la doctrine catholique. Des curés tentaient même d'influencer leurs ouailles en ce sens, du moins si l'on en croit un témoignage recueilli par la FNSJB selon lequel «au partir [sic] d'un sermon, qui laissait l'impression que la doctrine de l'Église s'oppose au suffrage féminin, l'on a fait signer une requête contre le vote des femmes ${ }^{85}$ 》.

Malgré toutes les résistances révélées en 1922 qui ont mené à la démission de Marie Gérin-Lajoie du Comité provincial du suffrage féminin, La Bonne parole continua à publier, assez régulièrement jusqu'en 1933, des articles favorables au mouvement suffragiste. La FNSJB ne cessa pas non plus d'adresser des félicitations aux députés qui présentaient les projets de loi visant à octroyer le droit de vote aux femmes ainsi que d'envoyer des représentantes à Québec pour les appuyer. Cela dément, ou du moins nuance, l'affirmation de Pinard selon laquelle la Fédération s'est retirée du dossier après $1922^{86}$. Bien entendu, en 1928, le désistement de MFr Gauthier ne facilita pas la position de la FNSJB. Tiraillée entre ses convictions politiques et religieuses qui s'avéraient de moins en moins conciliables, celle-ci a dû délaisser la participation active au débat pour se retrancher dans un appui écrit au mouvement et dans une ouvre d'éducation civique ${ }^{87}$. Dans La Bonne parole, on peut juger de l'influence du clergé sur les positions politiques de la Fédération:

Toujours prête à promouvoir la cause de la femme, la Fédération nationale Saint-Jean-Baptiste fut la première en tête du mouvement qui s'est dessiné, il y a quelques années, en faveur du suffrage féminin. [...] Son action dans ce domaine dut cependant se subordonner aux directions de l'épiscopat et, après avoir réclamé des parlements le droit de vote pour la femme, tendit, plutôt ensuite, à préparer celle-ci à exercer ce droit, en l'éclairant sur ses nouvelles responsabilités et en l'instruisant de ses devoirs civiques ${ }^{88}$.

85. ANQM, Fonds FNSJB, 06, MP/120, bobine 6823, procès-verbal du Bureau de direction de la FNSJB, 23 février 1922, 16.

86. On peut d'ailleurs reprocher à Pinard et à ses collègues de ne pas avoir poursuivi la recherche au-delà de 1922. Cette date ne correspond pas, à mon avis, à la fin de la participation de la FNSJB au dossier du suffrage féminin. Voir Yolande Pinard et al., «La Fédération nationale Saint-Jean-Baptiste...», 99.

87. AINDBC, Fonds de Marie Lacoste-Gérin-Lajoie, P2, boîte 47, dossier 17, lettre de Marie Gérin-Lajoie à Idola Saint-Jean, 7 novembre 1928. Il faut toutefois mentionner que jamais la Fédération n'a appuyé un parti politique. En cela, elle se distingue de l'Alliance canadienne pour le vote des femmes, dirigée par Idola Saint-Jean, et de la Ligue des droits de la femme présidée par Thérèse Casgrain. Ces deux organisations entretenaient des liens avec le Parti libéral du Québec.

88. Yvonne Letellier de Saint-Just, «À la veille du $25^{\mathrm{e}}$ anniversaire de la Fédération nationale Saint-Jean-Baptiste», $B P, 19,6$ (juin 1931): 7. 
Ce n'est toutefois qu'en 1933, c'est-à-dire après la retraite de Marie Gérin-Lajoie de la présidence de la Fédération, que le clergé a vraiment imposé ses vues au Bureau de direction de la FNSJB ${ }^{89}$. Mme Thibaudeau, qui la remplaça, n'avait jamais pris publiquement position au sujet du suffrage féminin et elle était particulièrement active au sein de l'Union internationale des ligues catholiques féminines qui prônait une totale obéissance au clergé local. Par ailleurs, la nouvelle vice-présidente, madame Brossard, avait de son côté fait ses preuves au sein de la Ligue des femmes chrétiennes, et elle s'était fait connaître par ses croisades au sujet de la pudeur vestimentaire des femmes ainsi que par ses positions calquées sur celles du clergé catholique canadien-français ${ }^{90}$.

Il n'en demeure pas moins que durant un bon nombre d'années, la Fédération nationale Saint-Jean-Baptiste, sous la gouverne de Marie Gérin-Lajoie, avait proposé une argumentation maternaliste en faveur du suffrage féminin. La différence entre les hommes et les femmes, la supériorité morale de ces dernières en raison de leur maternité potentielle, de même que la complémentarité naturelle et sociale des deux sexes constituaient les bases idéologiques sur lesquelles reposait l'argumentation de la Fédération qui s'est illustrée de façon convaincante dans ce dossier. La recherche et la revendication de certains droits au nom de la différence fondamentale des hommes et des femmes éloignent sans contredit la FNSJB du mouvement féministe, défini par la recherche de l'égalité et la contestation de la hiérarchie sexuelle établie.

Avec cet article, je voulais démontrer que le concept de maternalisme permet de faire la synthèse de l'orientation idéologique de la FNSJB. Les différents courants d'influence relevés il y a vingt ans par Yolande Pinard - le féminisme chrétien, le féminisme social et le féminisme de reven-

89. C'est peut-être ce qui explique le quasi-silence de la FNSJB en 1940 lorsque les femmes ont acquis le droit de vote. L'éditorial qui suit la décision de Godbout s'intitule «Notre champ d'action s'élargit», ce n'est donc pas le ton de la victoire tant attendue. On peut y lire: «Par cette loi, la femme acquiert un nouveau droit dans la province de Québec, mais elle assume aussi une nouvelle responsabilité.» Yvonne Letellier de Saint-Just, BP, 29,5 (mai 1940): 1.

90. En 1938, par exemple, lors d'un malentendu avec l'archevêché au sujet de l'appui de la FNSJB à des mémoires de l'Alliance canadienne pour le vote des femmes, madame Brossard, qui présidait l'assemblée du bureau de direction en l'absence de madame Thibaudeau, affiche ses couleurs en disant que «quelle que soit l'opinion personnelle de chacun des membres de la Fédération au sujet du suffrage féminin, la Fédération doit s'abstenir comme corps de s'aventurer sur ce terrain par obéissance aux directives de l'Autorité religieuse en cette matière», ANQM, Fonds FNSJB, 06, MP/ 120, bobine 6823, procès-verbal du Bureau de direction, 27 mai 1938, 9. 
dication juridico-politique - peuvent maintenant être intégrés à une catégorie d'analyse plus large basée sur la fonction maternelle des femmes. Cela me porte à distinguer entre féminisme et maternalisme, même si dans les faits les revendications concrètes de ces deux mouvements se sont souvent rejointes. La FNSJB s'insère ainsi dans la deuxième catégorie de mouvements féminins en particulier parce qu'elle a encouragé les femmes à mettre leur nature maternelle au service de la société, alors que les féministes ont plutôt revendiqué des droits démocratiques pour les femmes au nom de leur citoyenneté. Pour mener à bien ses revendications, la FNSJB a dû redéfinir les frontières entre le monde privé et le monde public en démontrant que l'évolution de la société avait transformé les relations entre ces deux mondes. Conséquemment, les femmes, pour être mères dans le plein sens du mot, se devaient d'élargir leur action à l'extérieur des murs de leur foyer. L'originalité de la Fédération repose beaucoup sur l'ampleur des revendications qu'elle a mises de l'avant en utilisant la fonction maternelle des femmes comme argument principal. J'ai soulevé ici le cas du suffrage féminin pour démontrer comment la FNSJB appliquait ses convictions idéologiques à des dossiers de revendications concrets. La lutte pour l'accès des femmes à l'éducation et à des emplois de qualité, la revendication pour l'amendement du code civil en faveur des femmes mariées et l'ensemble du dossier de la protection maternelle et infantile reposent sur les mêmes bases idéologiques et relèvent de la même argumentation maternaliste.

Des comparaisons avec d'autres organisations féminines québécoises pourraient permettre d'évaluer leur diversité idéologique et de situer plus précisément les positions de la FNSJB. Une étude de l'Alliance canadienne pour le vote des femmes, présidée par Idola Saint-Jean, qui semble être au premier abord beaucoup plus orientée vers des positions féministes et égalitaires, serait toute indiquée pour effectuer ce projet de comparaison. 